T. Watanabe

Nagoya Math. J.

Vol. 121 (1991), 195-199

\title{
ON THE STRONG UNIMODALITY \\ OF LÉVY PROCESSES
}

\author{
TOSHIRO WATANABE
}

\section{$\S 1$. Introduction and results}

A measure $\mu(d x)$ on $R$ is said to be unimodal with mode $a$ if $\mu(d x)$ $=c \delta_{a}(d x)+f(x) d x$, where $c \geqq 0, \delta_{a}(d x)$ is the delta measure at $a$ and $f(x)$ is non-decreasing for $x<a$ and non-increasing for $x>a$. A measure $\mu(d x)=\sum_{n=-\infty}^{\infty} p_{n} \delta_{n}(d x)$ on $Z=\{0, \pm 1, \pm 2, \cdots\}$ is said to be unimodal with mode $a$ if $p_{n}$ is non-decreasing for $n \leqq a$ and non-increasing for $n \geqq a$. A probability measure $\mu(d x)$ on $R$ (resp. on $Z$ ) is said to be strongly unimodal on $R$ (resp. on $Z$ ) if, for every unimodal probability measure $\eta(d x)$ on $R$ (resp. on $Z$ ), the convolution $\mu * \eta(d x)$ is unimodal on $R$ (resp. on $Z$ ). Let $X_{t}, t \in[0, \infty$ ), be a Lévy process (that is, a process with stationary independent increments starting at the origin) on $R$ (resp. on $Z$ ) with the Lévy measure $\nu(d x)$. The process $X_{t}$ is said to be unimodal on $R$ (resp. on $Z$ ) if, for every $t>0$, the distribution of $X_{t}$ is unimodal on $R$ (resp. on $Z$ ). It is said to be strongly unimodal on $R$ (resp. on $Z$ ) if, for every $t>0$, the distribution of $X_{t}$ is strongly unimodal on $R$ (resp. on $Z$ ). In this paper we shall characterize strongly unimodal Lévy processes on $R$ and $Z$.

Theorem 1. Let $X_{t}$ be a Lévy process on $R$. Then $X_{t}$ is strongly unimodal on $R$ if and only if

$$
X_{t}=\sigma B(t)+\gamma t,
$$

where $B(t)$ is a Brownian motion and $\sigma$ and $\gamma$ are constants, $\sigma \geqq 0$.

Theorem 2. Let $X_{t}$ be a Lévy process on $Z$. Then $X_{t}$ is strongly unimodal on $Z$ if and only if

$$
X_{t}=X_{a t}^{(1)}-X_{b t}^{(2)},
$$

Received June 25, 1990. 
where $X_{t}^{(1)}$ and $X_{t}^{(2)}$ are independent Poisson processes and $a$ and $b$ are non-negative constants.

Ibragimov [1] proves that a probability measure on $R$ is strongly unimodal if and only if it is a delta measure or absolutely continuous with support being an interval and the density being log-concave. As a counterpart on $Z$, Keilson-Gerber [2] proves that a probability measure $\mu(d x)=\sum_{n=-\infty}^{\infty} p_{n} \delta_{n}(d x)$ on $Z$ is strongly unimodal if and only if $p_{n}^{2} \geqq$ $p_{n+1} p_{n-1}$ for every $n \in Z$. These results play an essential role in our proof.

The following are main related results. Yamazato [9] shows that if the density of $|x| \nu(d x)$ is log-concave on $R-\{0\}$, then the distribution of $X_{t}$ is strongly unimodal on $R$ for sufficiently large $t>0$. It is an open problem to characterize unimodal Lévy processes on $R$ or $Z$ in terms of their Lévy measures. Wolfe [7] proves that, if $X_{t}$ is unimodal on $R$ (resp. on $Z$ ), then $\nu(d x)$ (resp. $\nu(d x)+c \delta_{0}(d x)$ for some $c>0$ ) is unimodal on $R$ (resp. on $Z$ ) with mode 0 , and that the converse does not hold. Medgyessy [3] shows that if $\nu(d x)$ is symmetric and unimodal on $R$, then $X_{t}$ is unimodal on $R$. The analogous result on $Z$ is observed by Wolfe [7]. As a big advancement, Yamazato [8] shows that Lévy processes of class $L$ are unimodal on $R$. Steutel-van Harn [4] proves the unimodality of Lévy processes on the non-negative integers analogous to class $L$. Watanabe [5] constructs non-symmetric unimodal Lévy processes on $R$ that are not of class $L$. Watanabe [6] gives a similar result for Lévy processes on the non-negative integers.

\section{§2. Proof of Theorem 1}

Let $\mu_{t}(d x)$ be the distribution of $X_{t}$. Then we have

$$
\begin{gathered}
\int_{-\infty}^{\infty} e^{i z x} \mu_{t}(d x)=e^{t \psi(z)}, \\
\psi(z)=i \gamma z-2^{-1} \sigma^{2} z^{2}+\int_{-\infty}^{\infty}\left(e^{i z x}-1-i z x\left(1+x^{2}\right)^{-1}\right) \nu(d x),
\end{gathered}
$$

where $\gamma \in R, \sigma^{2} \geqq 0$, and

$$
\nu(\{0\})=0 \text { and } \int_{-\infty}^{\infty} x^{2}\left(1+x^{2}\right)^{-1} \nu(d x)<\infty .
$$

The measure $\nu(d x)$ is called the Lévy measure of $X_{t}$. 
Proof of "if" part. Since normal distributions are strongly unimodal on $R$ by Ibragimov's result [1], $X_{t}=\sigma B(t)+\gamma t$ is strongly unimodal on $R$.

Proof of "only if" part. Suppose that $X_{t}$ is strongly unimodal on $R$ and not deterministic. Then, for each $t>0$,

$$
\mu_{t}(d x)=f_{t}(x) d x
$$

the set $\left\{x: f_{t}(x)>0\right\}$ is an interval, and $\log f_{t}(x)$ is concave on this set. This is by Ibragimov's result [1]. By Wolfe's theorem [7],

$$
\nu(d x)=\phi(x) d x
$$

with $\phi(x)$ non-decreasing for $x<0$ and non-increasing for $x>0$. It is well-known that, for any bounded continuous function $g(x)$ with support in $R-\{0\}$, it holds that

$$
\lim _{t \rightarrow 0} t^{-1} \int_{-\infty}^{\infty} g(x) \mu_{t}(d x)=\int_{-\infty}^{\infty} g(x) \nu(d x) .
$$

Hence, by Lemma 3 of Ibragimov [1], we can choose a sequence $t(n)$ such that, as $n \rightarrow \infty, t(n) \rightarrow 0$ and

$$
t(n)^{-1} f_{t(n)}(x) \longrightarrow \phi(x)
$$

for a.e. $x \in R$. It follows that $\log \phi(x)$ is concave on the support of $\phi(x)$ by (2.5). Therefore, $\phi(x)$ is bounded on $R$ and

$$
c=\nu(R)=\int_{-\infty}^{\infty} \phi(x) d x<\infty .
$$

Suppose that $c>0$. We shall show that this leads to a contradiction. Let

$$
\gamma_{0}=\gamma-\int_{-\infty}^{\infty} x\left(1+x^{2}\right)^{-1} \nu(d x)
$$

We can assume $\gamma_{0}=0$, because we can consider $X_{t}-\gamma_{0} t$ instead of $X_{t}$. There are two possible cases.

Case 1. $\sigma=0$. The process $X_{t}$ is a compound Poisson process and hence $\mu_{t}(\{0\})>0$. This is a contradiction because non-trivial strongly unimodal probability measure on $R$ has no point mass.

Case 2. $\sigma^{2}>0$. We get, for any $t>0$,

$$
\mu_{t}(d x)=\mu_{t}^{(1)} * \mu_{t}^{(2)}(d x),
$$


where $\mu_{t}^{(1)}(d x)=g_{t}(x) d x$ is the normal distribution with mean 0 and variance $\sigma^{2} t$, and $\mu_{t}^{(2)}(d x)$ is a compound Poisson distribution. Since $\mu_{t}^{(2)}(\{0\}) \rightarrow 1$ as $t \rightarrow 0$, we obtain from (2.7) that

$$
\lim _{t \rightarrow 0}\left\{g_{t}(0)\right\}^{-1} f_{t}(0)=\lim _{t \rightarrow 0}(2 \pi t)^{1 / 2} \sigma f_{t}(0)=1 .
$$

We have, by Ibragimov's theorem [1],

$$
\left\{f_{t}(x)\right\}^{2} \geqq f_{t}(0) f_{t}(2 x)
$$

for any $t>0$ and $x \in R$. Hence we obtain from (2.5), (2.8), and (2.9) that

$$
\begin{aligned}
0 & =\lim _{n \rightarrow \infty}(2 \pi)^{1 / 2} \sigma\{t(n)\}^{3 / 2}\left\{(t(n))^{-1} f_{t(n)}(x)\right\}^{2} \\
& \geqq \lim _{n \rightarrow \infty}(2 \pi t(n))^{1 / 2} \sigma f_{t(n)}(0)\left\{(t(n))^{-1} f_{t(n)}(2 x)\right\}=\phi(2 x)
\end{aligned}
$$

for a.e. $x \in R$.' It follows that $\phi(x)=0$ for a.e. $x \in R$. This contradicts the assumption $c>0$.

Therefore, if $X_{t}$ is strongly unimodal on $R$, then $\nu(d x)=0$. Thus we have proved Theorem 1 .

\section{§3. Proof of Theorem 2}

Let $X_{t}$ be a Lévy process on $Z$. Then we can write (2.1) as

$$
\psi(z)=\int_{Z}\left(e^{i z x}-1\right) \nu(d x)
$$

with $\nu(\{0\})=0$ and $\nu(Z)<\infty$.

Proof of "if" part. Since Poisson distributions are strongly unimodal on $Z$ by Keilson-Gerber [2], $X_{t}=X_{a t}^{(1)}-X_{b t}^{(2)}$ is strongly unimodal on $Z$.

Proof of "only if" part. Suppose that $X_{t}$ is strongly unimodal on $Z$. Let $\mu_{t}(d x)=\sum_{n=-\infty}^{\infty} p_{n}(t) \delta_{n}(d x)$ be the distribution of $X_{t}$. By KeilsonGerber's theorem [2], we have

$$
\left\{p_{1}(t)\right\}^{2} \geqq p_{0}(t) p_{2}(t)
$$

for any $t>0$. Since $\mu_{t}(d x)$ converges weakly to $\delta_{0}(d x)$ as $t \rightarrow 0$, we get

$$
\lim _{t \rightarrow 0} p_{0}(t)=1 \text {. }
$$

Since (2.4) holds, we have

$$
\lim _{t \rightarrow 0} t^{-1} p_{n}(t)=\nu(\{n\})
$$


for $n \neq 0$. Hence we obtain from (3.2), (3.3), and (3.4) that

$$
0=\lim _{t \rightarrow 0} t\left(t^{-1} p_{1}(t)\right)^{2} \geqq \lim _{t \rightarrow 0} p_{0}(t) t^{-1} p_{2}(t)=\nu(\{2\}) .
$$

Therefore we get $\nu(\{2\})=0$. Since $\nu(\{n\})$ is non-increasing for $n \geqq 1$ by Wolfe's theorem [7], this implies that $\nu(\{n\})=0$ for $n \geqq 2$. Similarly we have $\nu(\{n\})=0$ for $n \leqq-2$. The proof of Theorem 2 is complete.

\section{REFERENCES}

[1] Ibragimov, I. A., On the composition of unimodal distributions, Theor. Probability Appl., 1 (1956), 255-260.

[2] Keilson, J. and Gerber, H., Some results for discrete unimodality, J. Amer. Statist. Assoc., 66 (1971), 386-389.

[ 3 ] Medgyessy, P., On a new class of unimodal infinitely divisible distribution functions and related topics, Studia Sci. Math. Hungar., 2 (1967), 441-446.

[ 4 ] Steutel, F. W. and van Harn, K., Discrete analogues of self-decomposability and stability, Ann. Probability, 7 (1979), 893-899.

[5] Watanabe, T., Non-symmetric unimodal Lévy processes that are not of class L, Japan. J. Math., 15 (1989), 191-203.

[6] - On unimodal Lévy processes on the nonnegative integers, Preprint.

[7] Wolfe, S. J., On the unimodality of infinitely divisible distribution functions, Z. Wahrsch. Verw. Gebiete, 45 (1978), 329-335.

[8] Yamazato, M., Unimodality of infinitely divisible distribution functions of class L, Ann. Probability, 6 (1978), 523-531.

[9] — On strongly unimodal infinitely divisible distributions, Ann. Probability, 10 (1982), 589-601.

Nakamandokoro

Aizubangemachi

Fukushima-ken, 969-65

Japan 